University of Nebraska - Lincoln

DigitalCommons@University of Nebraska - Lincoln

$2-2002$

\title{
The Sequential Development of Jaw and Lip Control for Speech
}

Jordan R. Green

University of Nebraska-Lincoln, jgreen4@unl.edu

Christopher A. Moore

University of Washington, Seattle

Kevin J. Reilly

University of Washington, Seattle

Follow this and additional works at: https://digitalcommons.unl.edu/specedfacpub

Part of the Special Education and Teaching Commons

Green, Jordan R.; Moore, Christopher A.; and Reilly, Kevin J., "The Sequential Development of Jaw and Lip Control for Speech" (2002). Special Education and Communication Disorders Faculty Publications. 25. https://digitalcommons.unl.edu/specedfacpub/25

This Article is brought to you for free and open access by the Department of Special Education and Communication Disorders at DigitalCommons@University of Nebraska - Lincoln. It has been accepted for inclusion in Special Education and Communication Disorders Faculty Publications by an authorized administrator of DigitalCommons@University of Nebraska - Lincoln. 


\title{
The Sequential Development of Jaw and Lip Control for Speech
}

\author{
Jordan R. Green \\ Department of Communicative Disorders, University of Wisconsin-Madison \\ Christopher A. Moore \& Kevin J. Reilly \\ Department of Speech and Hearing Sciences, University of Washington, Seattle
}

\begin{abstract}
Vertical displacements of the upper lip, lower lip, and jaw during speech were recorded for groups of 1-, 2-, and 6 -year-olds and adults to examine if control over these articulators develops sequentially. All movement traces were amplitude- and time-normalized. The developmental course of upper lip, lower lip, and jaw control was examined by quantifying age-related changes in the similarity of each articulator's movement patterns to those produced by adult subjects and by same-age peers. In addition, differences in token-to-token stability of articulatory movement were assessed among the different age groups. The experimental findings revealed that 1- and 2year-old children's jaw movements were significantly more adult-like than their upper and lower lip movements, which were more variable. In contrast, upper and lower lip movement patterns became more adult-like with maturation. These findings suggest that the earliest stages of speech motor development are constrained by the nonuniform development of articulatory control, with the jaw preceding the lips. The observed developmental patterns suggest that the properties of the oral motor control system significantly influence the pattern of speech sound acquisition.
\end{abstract}

Keywords: speech development, motor development, lips, jaw, babble

The development of speech production ensues over an extended period and appears to significantly lag the attainment of many associated cognitive/perceptual capacities. Children typically do not master the sounds in their ambient language until 8 years old (Sanders, 1972); and some features of speech, such as formant frequencies and voice onset times, do not exhibit adult-like consistency until later (Eguchi \& Hirsh, 1969; Kent, 1976; Tingley \& Allen, 1975). The observation that the mastery of speech occurs over such an extended period suggests that infants are not endowed with the neuromuscular control for producing the range of sounds in their ambient language and, consequently, need to adopt strategies to approximate adult-like speech. These early ad- aptations provide a window into the developmental status of the neuromotor system and cognitive/perceptual processes. Careful study of these adaptations will be particularly useful for identifying the necessary precursors for speech, explaining across-speaker regularities in early phonologic development, and providing objectively based criteria for evaluating the developmental status of the oromotor system in children with suspected speech or feeding delays. Unfortunately, knowledge about the formation of articulatory gestures is limited because of the difficult task of acquiring articulatory data from young children, particularly those under the age of 4 years (Moore \& Ruark, 1996; Smith, Goffman, \& Stark, 1995). 
Predispositions observed in early speech suggest that young children have a propensity for certain articulatory configurations (i.e., "coordinative biases") and that the formation of articulatory gestures is guided by potent biologic constraints (Kent, 1976, 1992, 1999; Oller, 1980; Vorperian, Kent, Gentry, \& Yandell, 1999). Infants from different languages tend to produce a similar restricted set of speech sounds (Locke, 1983, 1993). Moreover, within a language system young children tend to acquire and master sounds in a similar order (Sanders, 1972; Stoel-Gammon, 1985) and use similar phonologic strategies while striving to produce intelligible speech (Preisser, Hodson, \& Paden, 1988). An improved understanding of speech development would benefit from more knowledge about the features of the immature oromotor control system that give rise to these tendencies in early speech development.

In the present investigation, we test the hypothesis that one fundamental bias in the development of speech is that control over the articulators develops sequentially. The specific sequence of emergence of articulatory control will influence (a) the short-term strategies adopted for approximating adult speech and (b) the long-term course of speech motor development. For instance, if control over the lips, tongue, jaw, velum, and larynx develops sequentially, young children would be obligated to rely on the "best suited" or most developmentally advanced articulator(s), with the less developed articulators contributing to a large portion of behavioral instability.

In general terms, the developmental sequence of a sensorimotor system reflects the developmental schedule of largely independent neural and anatomic mechanisms, with each component having a unique ontogenetic and phylogenetic history (Hall \& Oppenheim, 1987; Touwen, 1998). These conditions are particularly relevant to the developing oromotor system as the different articulators (a) are predominantly mediated by distinct neural centers (Barlow \& Farley, 1989; Kennedy \& Kuehn, 1989), (b) have unique biomechanic properties (Abbs, 1973; Ostry, Vatikiotis-Bateson, \& Gribble, 1997), and (c) experience different degrees of activity and use in early ontogeny depending on their actions during sucking, chewing, vocalizing, and breathing (Bosma, 1985; Green et al., 1997; Moore \& Ruark, 1996). In fact, the extent of functional partitioning within the neural centers controlling the articulators, especially in brainstem nuclei, underscores the distinctive ontogenetic and phylogenetic roles for these structures.

Careful study of fetal orofacial behaviors in a variety of mammals supports the suggestion that the morphologic and functional development of orofacial structures is asynchronous. Mandibular movement is observed before the full formation of lip musculature (Gasser, 1967; Humphrey, 1964). Moreover, the appearance of spontaneous oral movements in the fetus is orderly: jaw open- ing, jaw closing, tongue movement, and lip movement (see Herring, 1985; Humphrey, 1970, 1971). These findings must be interpreted with caution, however, because the influences of early spontaneous orofacial movements and early orofacial morphogenesis on speech motor development have not been firmly established.

A growing body of empirical evidence suggests that the mandible provides the fundamental patterns of early articulation that form the foundation for the learning of other, more specialized articulations (MacNeilage \& Davis, 1990a, 1990b, 2000; Nittrouer, 1993). Assigning a leading role for the mandible in early speech has significant consequences for theories of speech development and the practice of speech-language pathology, although to our knowledge this proposed sequence in early articulatory development has not been verified through direct study of articulatory movements or muscle activity patterns. Building on their acoustic-perceptual findings, MacNeilage and Davis (1990a, 1990b) developed the "frame/content" theory of early speech development, which proposes that mandibular-driven oscillations in vocal tract constrictions are the fundamental motor pattern for early speech. Similarly, in a detailed study of developmental changes in the acoustic features of speech, Nittrouer (1993) reported that acoustic features related to jaw gestures matured earlier than those related to tongue-body gestures.

The suggestion that control matures earlier for the jaw than for the lips has also received some physiologic support in several studies of lip and jaw kinematics of children 4 years old and up. On repeated trials of basic speech utterances, Sharkey and Folkins (1985) observed that 4-year-olds exhibited significantly greater variability in lower lip displacements than 7-year-olds; however, no difference was observed in the consistency of jaw displacements between these age groups. Smith (1995) examined developmental changes in the variability of lip and jaw displacements in 5-, 8-, and 11-yearolds and adults across a variety of phonemic contexts and also found that children's jaw displacements were less variable than their lip displacements.

Additional evidence for the advanced development of jaw control in early speech comes from a previous investigation in which we reported the jaw to be the prime mover in early articulations, with the contribution from lower lip increasing gradually between ages 1 and 6 years old (Green, Moore, Higashikawa, \& Steeve, 2000). However, because our analyses were not designed to characterize the stability and form of early articulatory movement patterns, we could not determine whether the observed large-amplitude jaw movements represented a source of stability or instability in early speech. Fortunately, basic speech utterances, such as a /baba/, provide an easily identifiable developmental target for gauging the stability of early articulatory control because they are within the infant's vocal repertoire, and 
articulatory movement patterns for these utterances are remarkably similar in topology across adult speakers (Green et al., 2000). In this follow-up study we compare adults' upper lip, lower lip, and jaw movement patterns to those of children of differing ages to test for differences among the articulators in which they obtain the mature movement pattern. These data will be used specifically to test the hypothesis that jaw movement patterns exhibit early stability relative to upper and lower lip movement patterns.

\section{Method}

\section{Subjects}

Thirty-four subjects made up four subject groups: 5 infants (three females and two males; age range: 11 to 13 months, SD: 1), 9 toddlers (four females and five males; age range: 23 to 29 months, SD: 3), 10 children (gender balanced; age range: 6 to 7 years, SD: 3), and 10 adults (gender balanced; age range: 27 to 35 years, SD: 4). Complementary kinematic analyses were reported previously from these subjects (Green et al., 2000). Seventeen additional subjects (15 infants and 2 two-year-olds) failed to produce the target utterances during the experiment. This large number of unsuccessful data collection sessions was expected for these young subjects because (a) their verbal imitation skills varied, (b) the period of observation was relatively short ( $45 \mathrm{~min}-1 \mathrm{hr}$ ), and (c) the target utterances were narrowly specified. All participants were native speakers of American English. Before acceptance into the study, potential subjects were prescreened by interview of either the adult subject or the child's parent for positive histories of developmental or neurological disorders and speech, language, hearing, or vision problems.

\section{Speech Samples}

Upper lip, lower lip, and jaw movements were analyzed during productions of baba, papa, and mama. These speech utterances were selected because mature speakers produce them with a high degree of consistency on repeated trials (Green et al., 2000), which provided an easily identifiable prototype that could be used to gauge the maturity of articulatory movements from children of differing ages. Moreover, infants spontaneously and imitatively produce baba and mama in isolation or in canonical babble sequences. Before analysis, each production was judged by the transcriber to be an acceptable bilabial CVCV utterance based on the video image and audio signal. In addition, only utterances produced with complete lip closure, as observed on a video recording, were included in the analysis. Utterances associated with "normal" dysfluencies (i.e., block or hesitation), coughs, or mispronunciations were not included in the study.
A total of 726 utterances were included in the analysis ( 1-year-olds, 35 utterances; 2-year-olds, 174 utterances; 6-year-olds, 254 utterances; adults, 263 utterances). Adult and 6-year-old subjects read the target words from a poster at a normal conversational rate and loudness in a pseudorandom order. These subjects repeated each word 5 to 10 times, yielding between 15 and 30 samples per subject for each utterance type. One-and 2-year-old subjects' productions were produced spontaneously or elicited through imitation. Speech samples from these subjects were elicited during play involving the child, the caretaker, and the experimenter. Because vocal imitation is inconsistent at these ages, 1- and 2-year-old subjects produced only a subset of the target utterances.

\section{Data Collection and Recording Conditions}

Lip and jaw movements were captured using a monochrome camera (Burle, TC351A) coupled to a videorecorder (Panasonic, AG-1980). As displayed in Figure 1, three single, flat, circular reflective markers (Is similar to $2 \mathrm{~mm}$ in diameter) were placed at the midline on the margin of the vermilion border of the upper lip (UL), lower lip (LL), and just superior to the mental protuberance of the mandible (J). Two reference markers (Is similar to $2 \mathrm{~mm}$ in diameter), also placed midline, one on the tip of the nose and one on the nasion, were used to correct for head movement. These two markers translated the origin to the nasion marker and aligned the axis to the line defined by these two markers. A reference marker, placed on the subject's forehead, was used to calibrate the displacement signals. The reflective markers were illuminated with an infrared light source. Subject's utterances were recorded using a wireless remote microphone (Telex, FMR-25) coupled to a digital audio recorder (Panasonic, SV-3700).

\section{Digitization, Signal Conditioning, and Normalization}

Target utterances were identified on the continuous video recordings and digitized into separate files. The vertical positions of the upper lip, lower lip, and jaw were extracted automatically from each digitized video file using a computer-based movement tracking system (Motus, version 2, Peak Performance). We have assessed the accuracy of this movement tracking system to be better than $.1 \mathrm{~mm}$ under these experimental conditions (Green et al., 2000). Subsequent to movement tracking, the displacement signals were digitally low-pass filtered $\left(\mathrm{f}_{1 \mathrm{p}}=\right.$ $15 \mathrm{~Hz}$ ) using a zero-phase shift forward and reverse digital filter (Butterworth, 8 pole). The lower-lip signal was derived by subtracting the lower-lip displacement signal from the jaw signal. An example of a kinematic record from an adult subject is presented in Figure 2. 


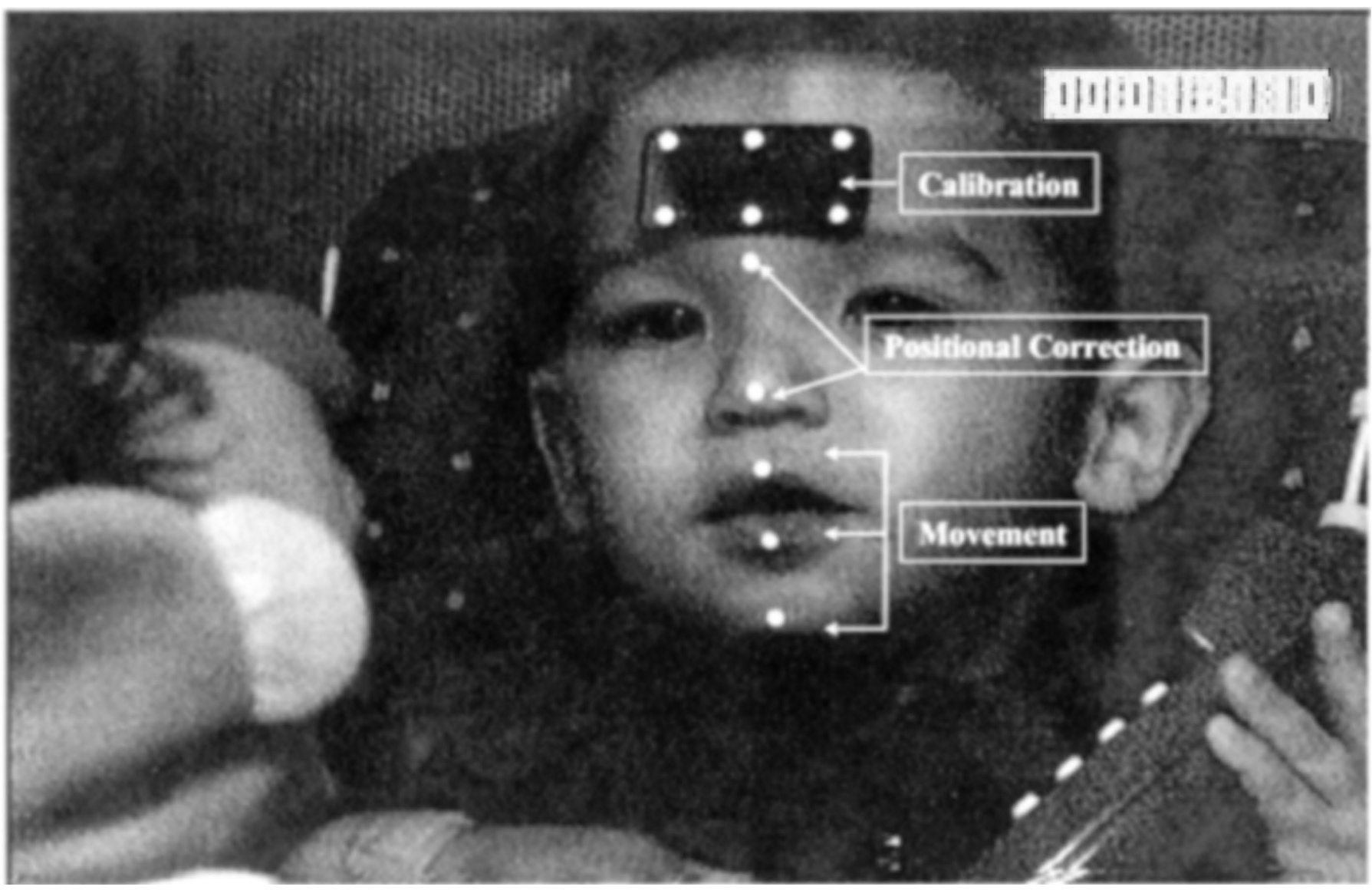

Figure 1. A 2-year-old subject fitted with calibration and movement markers for upper lip, lower lip, and jaw.

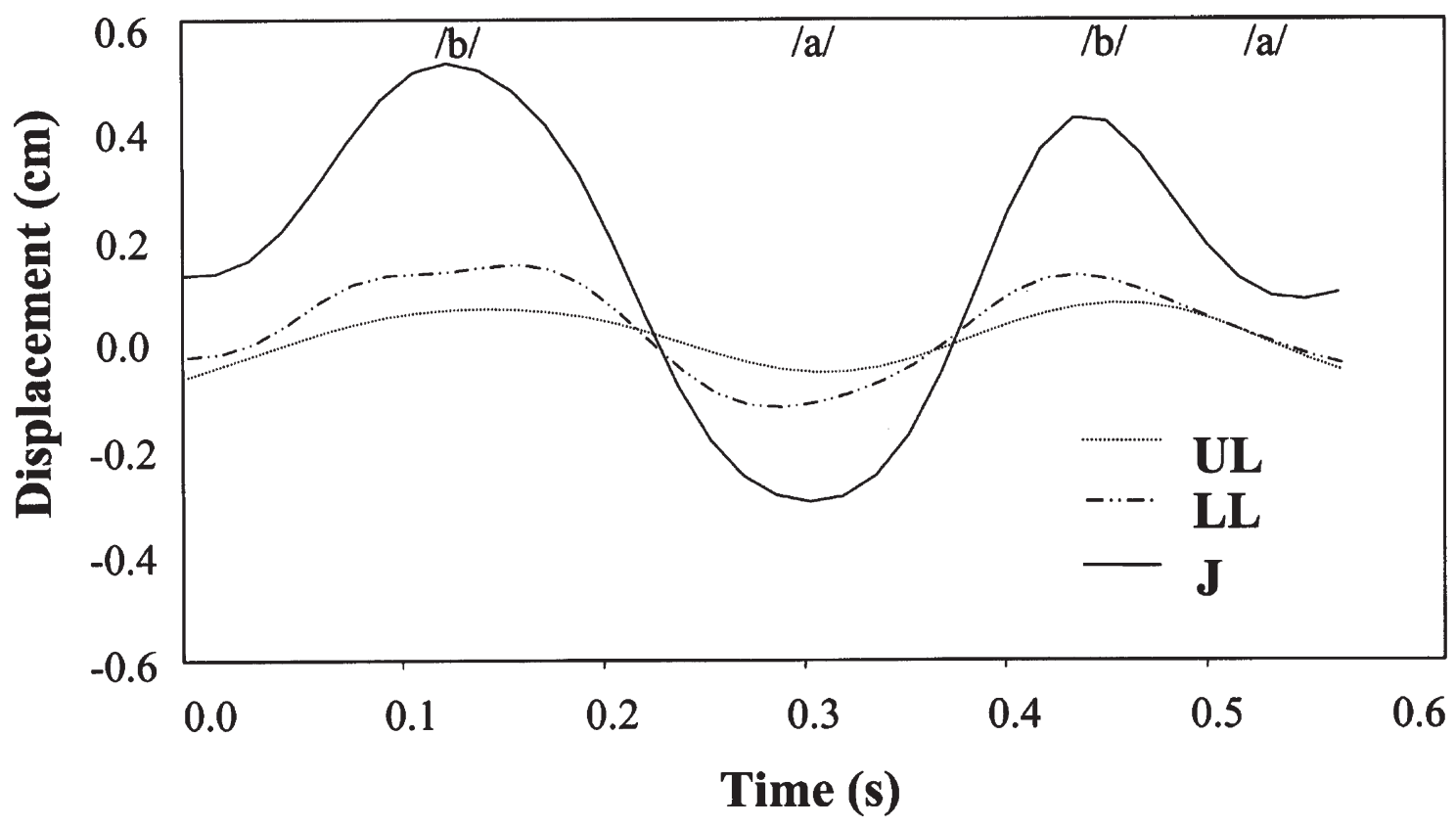

Figure 2. The treated kinematic traces from upper lip (UL), lower lip (LL), and jaw (J) produced by an adult subject saying baba. For ease of interpretation, each signal has been centered about its mean and the UL signal has been inverted. 
The onset and offset of UL, LL, and J movements associated with each utterance were estimated using the jaw's velocity trace (first-order derivative of the displacement signal). The jaw signal and its velocity trace were displayed on a computer monitor. Near-zero crossings $(-0.03 \mathrm{~mm} / \mathrm{s})$ were identified on each derived velocity trace. The $-0.03 \mathrm{~mm} / \mathrm{s}$ threshold was empirically derived and was adopted to ensure that the selected segments were associated with speech movements as opposed to those associated with small amplitude fluctuations in position that occur frequently at rest, especially in young children. The experimenter was required to visually confirm the algorithm's estimate of each boundary. Segmentation of the upper lip, lower lip, and jaw signals associated with each utterance was based solely on the jaw velocity signal because identification of kinematic events in the upper lip and lower lip signals was unreliable in young subjects. The data that could be yielded from canonical babble was maximized by parsing them into two-syllable segments, with adjacent segments overlapping by one syllable. For exam- ple, a $\mathrm{CV}_{1} \mathrm{CV}_{2} \mathrm{CV}_{3}$ was parsed into two utterances such that the $\mathrm{CV}_{2}$ occupied the final syllable of the first utterance $\left(\mathrm{CV}_{1} \mathrm{CV}_{2}\right)$ and the initial syllable of the second utterance $\left(\mathrm{CV}_{2} \mathrm{CV}_{3}\right)$. There were only three instances of canonical babble that exceeded two syllables (one threesyllable and two four-syllable).

All movement traces were amplitude and time normalized using methods similar to those previously described by Smith and colleagues (Goffman \& Smith, 1999; Smith \& Goffman, 1998). Amplitude normalization was achieved by dividing each movement trace by its standard deviation. Subsequently, linear temporal normalization was achieved by interpolating each signal to 1000 points using a commercially available cubic spline fit algorithm (Matlab, version 5.2). Spatiotemporal normalization of the signals provided a means to examine changes in relative time while minimizing variation from rate and absolute amplitude of movement across and within speakers. Figure 3 displays the raw and normalized traces for the upper lip, lower lip, and jaw from 10 repetitions of baba produced by one of the adult subjects.
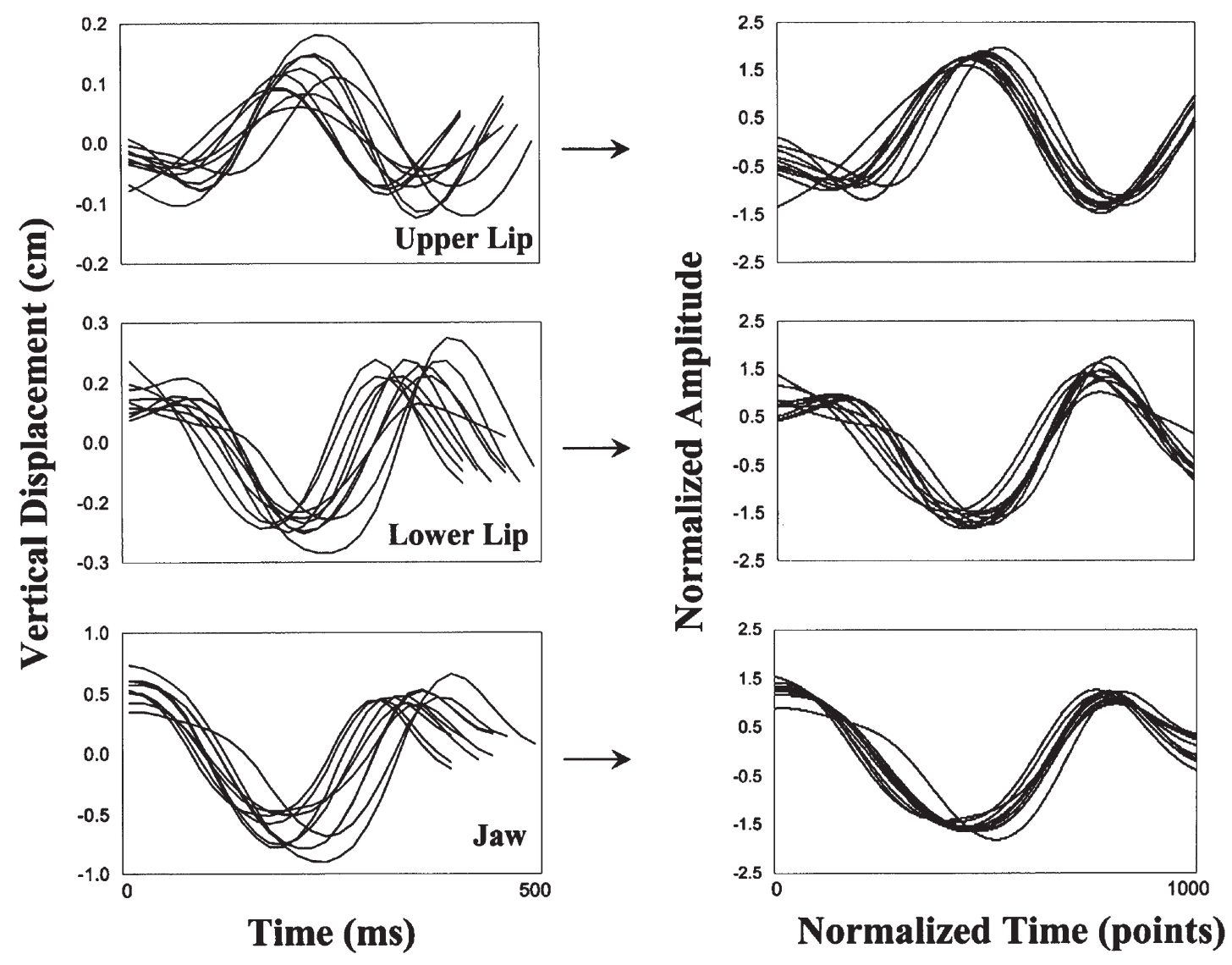

Figure 3. The left panel displays 10 kinematic records based on 10 repetitions of baba for the upper lip, lower lip, and jaw. The right panel shows alignment of the signals following the spatial and temporal normalization procedure. The high degree of token-to-token stability exhibited by this subject was typical for adult subjects. 


\section{Quantitative Analyses of the Kinematic Traces}

Differences in the developmental course of upper lip, lower lip, and jaw control were examined by quantifying the similarity among each subject's movement traces to three average signals using zero-lag cross-correlations. Each subject's upper lip, lower lip, and jaw traces were correlated with an (a) average adult trajectory (across-age comparison), (b) average within-age trajectory (within-age comparison), and (c) average withinsubject trajectory (within-subject comparison). In the present context, the term trajectory refers to the vertical time-history of each marker. These average trajectories were computed independently for the upper lip, lower lip, and jaw. The average adult trajectory was computed by averaging the average normalized traces produced by each adult subject. The average within-age trajectory was computed independently for each subject by averaging the average normalized trajectories produced by each subject within the age group while excluding his or her data from the computation. For adult subjects only, the average within-age and average across-age trajectories were inherently the same signals. Finally, the average within-subject trajectory was computed for each subject based on his or her normalized traces. All averaging was only within articulator.

One potential problem associated with parsing the lip displacement signals based on the jaw signal is that asynchronies between their movements will reduce the strength of correlation among the grouped lip signals, especially if asynchrony varies across trials. In a previous investigation we reported that the average lag between lip and jaw movements within a trial was relatively short, less than $30 \mathrm{~ms}$, for the same bilabial utterances (Green et al., 2000, Figure 8). Moreover, the average lag between upper lip and jaw was observed to decrease with age, reducing in duration from 18 to $29 \mathrm{~ms}$ between age 1 year and adulthood. To examine the influence of movement asynchrony on correlation strength, we correlated two $2.5 \mathrm{~Hz}$ sine waves with one signal lagging the other by $30 \mathrm{~ms}$. These signals were similar in length and duration to the present kinematic data $(1.5$ cycles in $608 \mathrm{~ms}$, sampling interval $=0.016)$. As in the present analysis, the signals were interpolated to 1000 points before being correlated. The derived coefficient for the lagged signals was 0.92, indicating that if lip signals vary by as much as $30 \mathrm{~ms}$, correlation coefficients for these data will be decreased by 0.08 . Given this result we judged the magnitude of potential asynchrony effects to be small relative to the differences in coefficient values across articulators that are observed in this study.

Because none of the 1-year-olds produced utterances that contained a [p] exemplar and half of the 2-year-olds produced five or fewer of these utterances, the adult and 6-year-old data were examined for phoneme effects before analysis of the entire data corpus. The results of a one-way analysis of variance (ANOVA) performed on the 6-year-olds' and adults' Fisher's $z$ transformed coefficients indicated that there were no statistically significant phoneme effects for each experimental condition (i.e., across-age, within-age, and within-subject). Given this finding, each subject's transformed coefficients were collapsed across phonemes to yield a single average for each comparison.

\section{Interpretation of Quantitative Methods}

In these analyses, where each subject's trajectories were correlated with the three average trajectories, coefficient values approaching 1.0 indicated that the subject's trajectories shared similar spatiotemporal characteristics with the average trajectory. Coefficients approaching zero indicated that the trajectories differed substantially in their spatiotemporal attributes. Negative coefficients indicated that the trajectories tended to move in opposite directions.

The comparison of each subject's trajectories with the average adult trajectory (across-age comparison) provided a means to test for across-articulator differences in the attainment of the mature movement pattern. In this analysis, the adult pattern represented the developmental target for this movement sequence. Because there are a number of explanations for why young children's movement patterns would correlate poorly with those of adults, examination of within-age and within-subject comparisons provides a context for interpreting the findings from the comparison with the mature movement patterns (across-age). Specifically, the within-age comparison distinguishes if differences among age groups arise from within-age differences (i.e., relatively low coefficient values in the within-age comparison with relatively low values in the across-age comparison) or from age-related differences in movement patterns (i.e., relatively high coefficients values in the within-age comparison with relatively low coefficient values in the across-age comparison). This multilevel analysis also provides an examination of the extent of age-related stages in articulatory development. For example, age-related stages in the development of articulatory coordination would be supported if coefficient values were significantly higher for within-age comparisons than for across-age comparisons.

The within-subject comparison facilitated interpretation of the across-age findings by distinguishing those differences among the age groups arising from movement instability (i.e., relatively low coefficient values in the within-subject comparison, with commensurately low coefficient values in the across-age comparison) from those arising from age-related differences in movement 
patterns (i.e., relatively high coefficient values in the within-subject comparison and low values in the acrossage comparison). The within-subject comparison also provided a metric for grossly quantifying developmental changes in movement pattern stability as a function of articulator.

\section{Statistical Analysis}

The Fisher's $z$ transformed coefficients from each subject were collapsed and averaged for each condition to yield 9 scores for each subject: 3 levels of articulator (upper lip, lower lip, jaw), 2 levels of comparison (across articulator, within articulator), and 3 levels of analysis (across age, within age, within subject). Each subject's averages were entered into multiple Bonferroni corrected $t$ tests to test for differences in the developmental status among articulators within and across levels of analysis. Because a Bonferroni adjustment based on all pairwise comparisons would have resulted in an excessively conservative criterion, alpha levels were adjusted separately for the within- and across-articulator comparisons. At each level, there were 18 within-articulator/across-age comparisons (adjusted $a=0.003$ ) and 12 across-articulator/within-age comparisons (adjusted $=0.004)$. There were 24 additional across-level-of-analysis comparisons (i.e., a comparison between the 1-yearolds' scores for jaw in the within-subject analysis to their scores for jaw in the within-age analysis), with an alpha level of 0.002. Due to the large number of comparisons being tested, statistical reporting is abbreviated throughout such that only criterion alpha levels are reported with significant results. In cases where the same criterion applied to multiple comparisons, the form " $p$ $<0.003$, for each comparison" is used.

\section{Results}

\section{Similarity to Mature Movement Patterns: Across-Age Comparison}

Maturation of articulatory movement patterns was quantified by correlating (zero-lag cross-correlations) each subject's movement trajectories with the average adult movement trajectory. The inset in Figure 4 compares a 1-year-old's signals to those of the average adult trajectory. The results in Figure 4 suggest that each articulator has a unique developmental schedule, with the jaw attaining the mature movement pattern much earlier than the upper and lower lip. Across all articulators, average coefficient values varied widely, ranging from 0.36 to 0.96 .

Because the adult subjects uniformly produced smooth and stereotyped kinematic profiles, they exhibited high coefficient values for all articulators and no articulator effects. In contrast, 1- and 2-year-olds' coefficients for jaw signals were significantly greater than were those for upper and lower lip $(p<0.004$ for each comparison). For 2-year-olds, coefficients for lower lip were significantly greater than those for upper lip $(p<$ 0.004 for each comparison). As indicated by the error bars in Figure 4, variability among subjects was notable for upper and lower lip at these ages. Six-year-olds produced articulatory movements similar to those of the adults, as indicated by the high coefficient values for all articulators. However, unlike adult subjects, articulator effects were observed for 6-year-olds, with the jaw exhibiting significantly greater similarity to the adult's average trace than upper and lower lip $(p<0.004$ for each comparison).

Coefficients for jaw movement were relatively high for all age groups, ranging from 0.63 to 0.97 . Across age groups, coefficients associated with jaw movements were significantly lower for 1- and 2-year-olds than for 6 -year-olds and adults ( $p<0.003$ for each comparison). One- and 2-year-olds' coefficients for lower lip were significantly lower than those exhibited by 6-year-olds and adults ( $p<0.003$ for each comparison). The degree of variation among 1-year-old subjects in their lowerlip coefficients was considerable, as indicated by the SD bars in Figure 4. Across age groups, coefficients associated with upper-lip movements were significantly lower for 1- and 2-year-olds than for 6-year-olds and adults ( $p<0.003$ for each comparison). The upper lip developmental pattern paralleled that observed for the lower lip.

\section{Age-Dependent Lip and Jaw Movement Pat- terns: Within-Age Comparison}

In this analysis, each subject's signals were compared to a trajectory that was derived by averaging all of the subjects' traces that were within his or her age group. Thus, coefficient values in this analysis represent the degree of similarity of movement patterns among sameage subjects. As exhibited in Figure 5, developmental differences were also revealed in the within-age-group comparisons of upper lip, lower lip, and jaw movement patterns. The inset in Figure 5 compares a 2-yearold's upper lip, lower lip, and jaw signals with average trajectories.

The existence of age-based phases in motor development would be supported if the coefficient values observed in this comparison were significantly greater than those in the across-age comparison, suggesting that children in each age group produced movement patterns that were more similar to those of same-age peers than to those of adults.

Within-age similarity for jaw was uniformly high for all the age groups, indicating a high degree of sim- 
ilarity of mandibular movement patterns among sameage peers. In contrast, the coefficients associated with upper lip and lower lip movements were initially low and increased with age ( $p<0.003$ for each comparison). Lower-lip coefficient values increased significantly between 1 and 2 years old and between 2 and 6 years old $(p<0.003$ for each comparison). For upper lip, the similarity of movement patterns within each age group was significantly less for 1- and 2-year-old subjects than for adult subjects ( $p<0.003$ for all), but was not significantly different among any of the child groups. As a group, 6year-old subjects produced similar movement patterns for lower lip and jaw, as indicated by the uniformly high coefficients exhibited for these articulators at this age.

The existence of age-dependent lip and jaw movement patterns was supported by differences between the coefficient values in the within-age (Figure 5) and acrossage (Figure 4) comparisons for 1- and 2-year-old subjects. Two-year-olds produced jaw and lower lip movement patterns that were more similar to those of same-age peers than to those of adults $(p<0.002$ for each comparison). In addition, 1- and 2-year-old subjects exhibited significantly greater coefficient values for the within-age comparison than for the across-age comparison for upper lip ( $p<0.002$ for each comparison).

\section{Movement Stability on Repeated Trials: Within-Subject Comparison}

Figure 6 shows the within-subject coefficient values for the upper lip, lower lip, and jaw as a function of age. In this analysis, the subjects' traces were compared to an average trajectory that was based solely on their individual traces. The inset in Figure 6 compares a 2-year-old's upper lip, lower lip, and jaw traces to average trajectories. These coefficient values reflect the degree of consistency in articulatory performance on repeated trials. The within-subject coefficients associated with the jaw were uniformly high for all age groups. In contrast, consistency of lower lip performance was significantly less

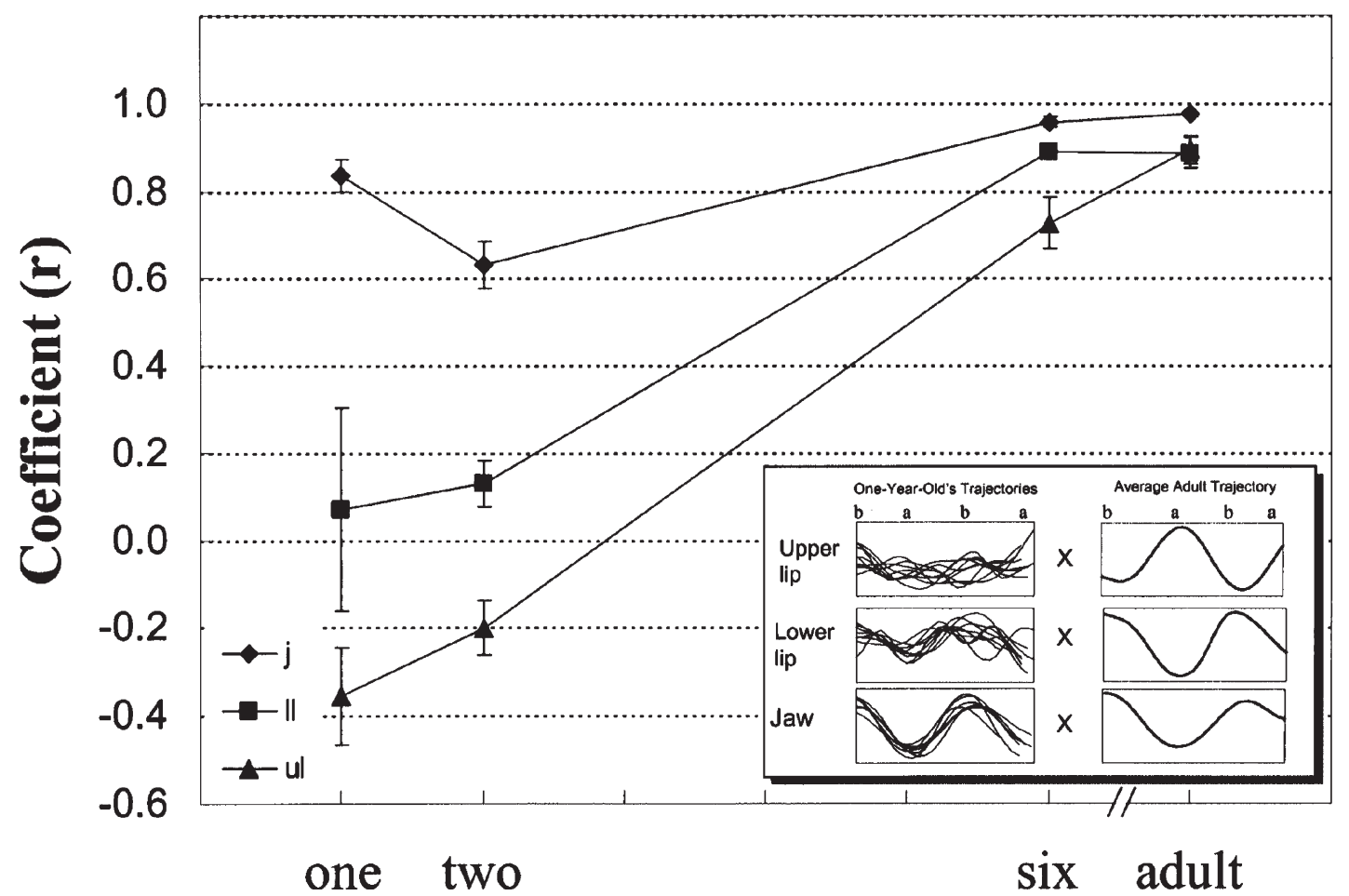

Age Group

Figure 4. In the across-age comparison, each subject's kinematic traces were correlated with an average trajectory computed on the adult subjects' traces. Data points in this figure represent the average coefficient values across subjects within each age group. All coefficients were transformed using the Fisher's $z$ before averaging and analysis. In this figure, the inverse transform was applied to each average for display purposes. The inset displays the kinematic traces from a 1-year-old subject for the upper lip (UL), lower lip (LL), and jaw (J) and the average adult trajectory that each trace was correlated with. A separate average trajectory was computed for each adult subject by removing his or her data from the computation. Error bars represent standard error among subjects in each age group. 
in 1- and 2-year-old subjects than in adults $(p<0.003$ for each comparison). Age comparisons also revealed that 2-year-olds' upper lip movement patterns were significantly less stable across repetitions than those produced by 6-year-olds and adults $(p<0.003$ for each comparison). However, 1-year-olds' upper-lip coefficient values did not differ significantly from those produced by 2 and 6-year-olds and adults.

The results of the within-subject comparison provided an additional context for interpreting the results of the across- and within-age comparisons. For instance, low coefficient values in the across- and within-age comparisons might be the result of movement instability, which will be revealed as low coefficients in the within-subject comparison. In contrast, the observation of relatively high coefficient values in the within-subject comparisons would suggest that any observed differences in the across-and within-age comparisons are due primarily to qualitative differences in movement patterns and not to overall movement instability.
Jaw coefficient values were not statistically different across any level of comparison. In contrast, lower lip coefficients were (a) greater in the within-subject comparison than in the across- and within-age comparisons for 1-year-olds and (b) greater in the within-subject comparison than in the within-age comparison for 2-year-olds ( $p<0.002$ for each comparison). For 1- and 2-year-old subjects, upper lip coefficient values were significantly greater in the within-subject comparison than in the across-age comparisons ( $p<0.002$ for each comparison).

\section{Discussion}

The present findings suggest that upper lip, lower lip, and jaw have distinct developmental schedules, with mature movement patterns for speech emerging earlier in the mandible than in the upper and lower lips. These data provide some physiologic support for the suggestion that early jaw movement patterns provide the foun-

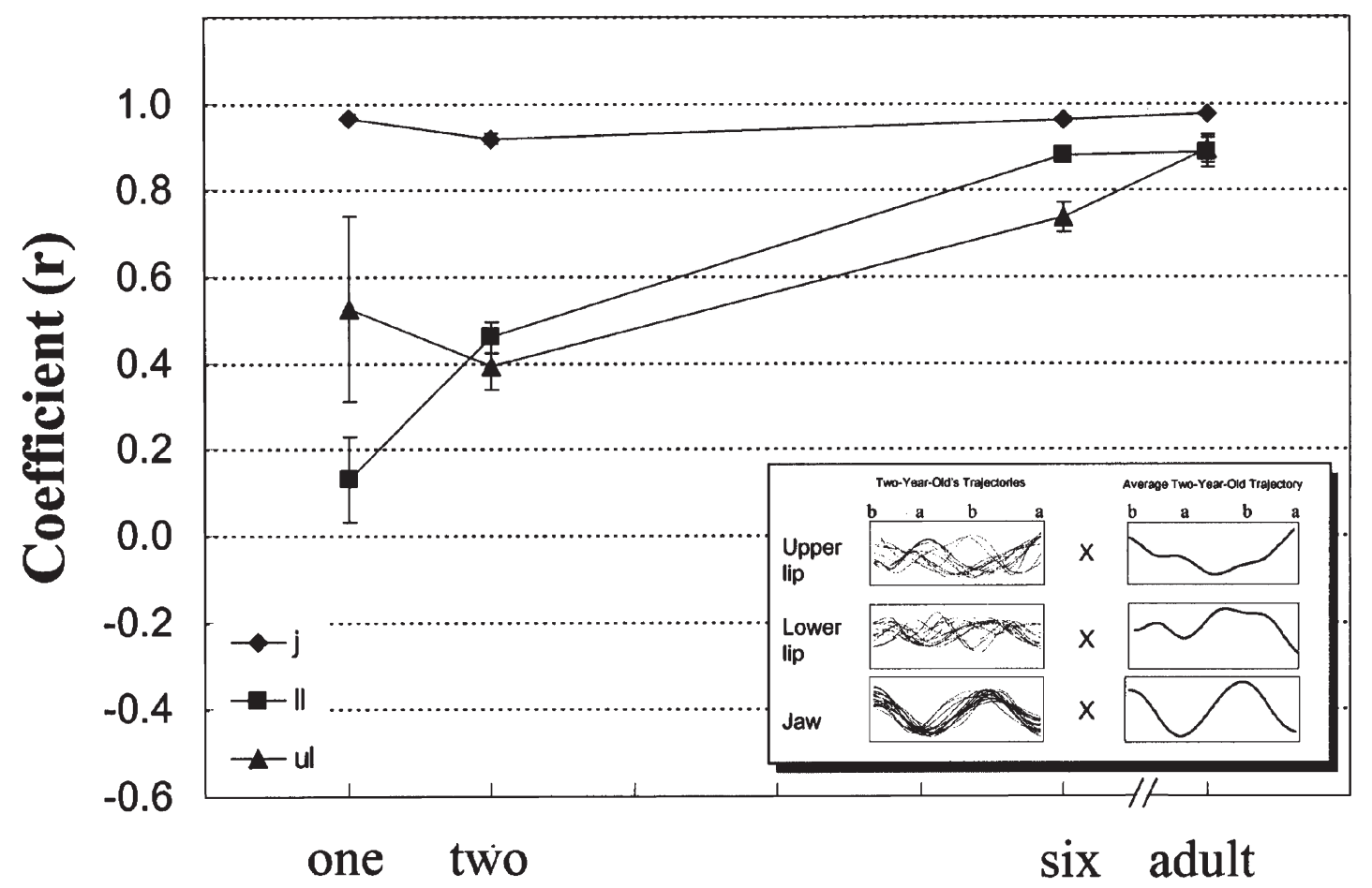

Age Group

Figure 5. In the within-age comparison, each subject's kinematic traces were correlated with an average trajectory computed on the traces produced by subjects in the same age group. Data points in this figure represent the average coefficient values across subjects within each age group. All coefficients were transformed using the Fisher's $z$ before averaging and analysis. In this figure, the inverse transform was applied to each average for display purposes. The figure inset displays the kinematic traces from a 2-year-old subject and the average 2-year-old trajectory that the traces were correlated with. A separate average trajectory was computed for each subject by removing his or her data from the computation. Error bars represent standard error among subjects in each age group. 
dation for the acquisition of other, more specialized motor skills that require integration of lower lip and tongue (MacNeilage \& Davis, 1990a, 1990b). This proposed developmental sequence has predictable consequences for the sequence of sound acquisition. For instance, young children's reliance on the mandible to approximate adult-like speech targets will constrain their phonetic inventory and predispose them to produce predictable speech errors and distortions. Collectively, the present findings suggest that (a) some components of articulatory gestures are organized, rather than highly variable, uncoordinated sensorimotor acts and that (b) articulatory ensembles form in the background of the unique developmental courses of individual articulators.

\section{Development of Articulatory Control Is Sequential}

During the first years of life, the jaw "outperformed" the upper and lower lip in all measures examined. For these simplistic speech utterances, 1-year-olds' jaw movements were remarkably similar to those of the adults. In contrast, 1-year-olds' upper and lower lip movement patterns did not resemble the adults' and exhibited high across-subject variability. Moreover, as indicated by the average coefficient values presented in Figure 6, although jaw movement patterns were relatively stable at 12 months old and were not associated with significant developmental changes, lip movement patterns were initially very unstable and became significantly more consistent with age (decreases in withinsubject variability). These results are in contrast to those exhibited by the mature speakers, who produced high degrees of movement stability for all articulators within and across speakers. Based on these observations, it appears that speech development involves integrating lip movement into a relatively well-established mandibular movement pattern. A leading role for the jaw in early speech has been implied in clinical models of early speech development (Hayden \& Square, 1994) and studies based on acoustic/ perceptual analysis of early speech

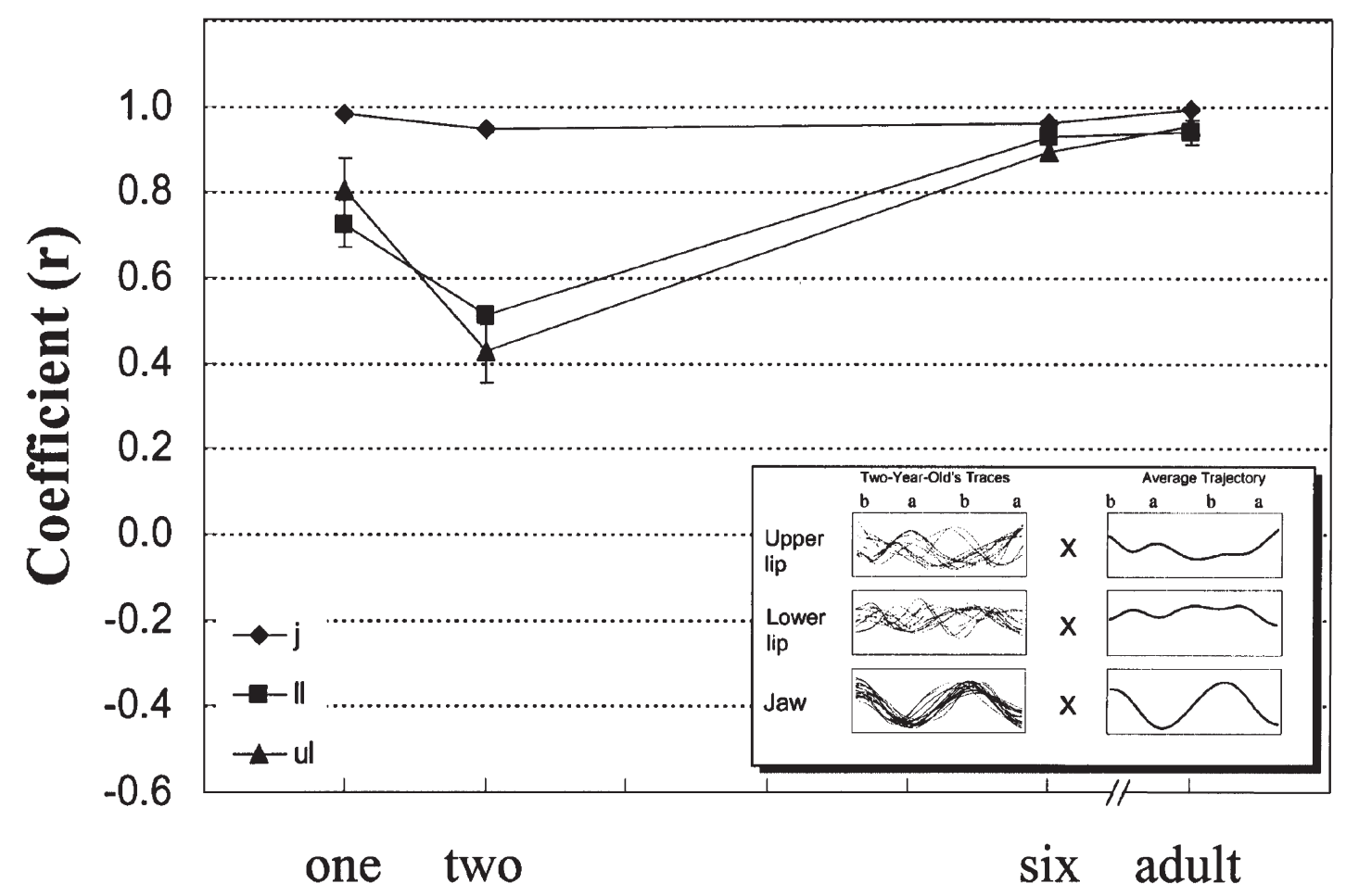

Age Group

Figure 6. In the within-subject comparison, each subject's kinematic traces were correlated with an average trajectory that was based on his or her individual traces. Data points in this figure represent the average coefficient values across subjects within each age group. All coefficients were transformed using the Fisher's $z$ before averaging and analysis. In this figure, the inverse transform was applied to each average for display purposes. The figure inset displays the kinematic traces produced by a 2-year-old subject and the computed average that the traces were correlated with. Error bars represent standard error among subjects in each age group. 
(MacNeilage \& Davis, 1990a; Nittrouer, 1993), but articulatory data from young children to support this suggestion has previously been lacking.

One unexpected finding was that 2-year-olds' jaw movements correlated less with the adults' than the 1year-olds' jaw movements did (Figure 4). Sussman and colleagues (Sussman, Minifie, Buder, Stoel-Gammon, \& Smith, 1996) reported a similar increase in variability in their acoustically derived parameters of coarticulation (i.e., locus equation parameters) between the ages of 12 and 21 months. These authors speculated that the increase in variability observed at age 21 months reflected not only the "added complexity of controlling independent articulator" but also inexperience with "specific, lexically driven, phonemic targets ..." (p. 431). The present findings that 2-year-olds' jaw movements exhibited decreased "maturity" (Figure 4) and stability (Figure 6) strengthens Sussman and colleagues' (1996) assertion that young children's articulatory systems may be particularly unstable during the early stages of lexicon expansion, which occurs between ages 1 and 2 years.

In the present investigation, adult speakers combined upper lip, lower lip, and jaw motions to achieve bilabial closure. Because young children have the potential to vary the relative contributions from each of these articulators for oral closure, we anticipated greater variation within and across children in the strategies they adopted to produce these bilabial utterances. For instance, young children could have fixed the position of the mandible while moving the lips to reduce the number of elements to be controlled. Additionally, the control of individual articulators could have coemerged, developing in parallel. The finding that children exhibit a relatively uniform strategy that relied on mandibular movement suggests that the immature orofacial motor system is biased, with the mandible having a distinct developmental advantage over the lips for speech.

\section{Early Speech Exhibits Some Highly Stable Movement Patterns}

The within-subject analysis provided a means to examine the degree of organization in early articulatory control. In this analysis, coefficient values approaching 0 were interpreted to suggest that young children's movements are highly variable, and coefficient values approaching 1 were interpreted to suggest that young children's movements are stable across repeated trials. For the within-subject analysis, coefficient values were relatively high for all age groups (Figure 6) in comparison to those associated with the across-age (Figure 4) and withinage (Figure 5) comparisons. Elevated coefficient values in the within-subject comparison were anticipated based on the expectation that articulatory movement patterns will contain subtle features that are unique to each speaker.
The relatively moderate degree of token-to-token stability observed in our younger subjects parallels the repetitive, stereotyped behaviors reported widely in early ontogeny in humans and other species (rat: Bekoff \& Trainer, 1979; chick: Hamburger \& Oppenheim, 1967; human: Meier, McGarvin, Zakia, \& Willerman, 1997; Thelen, 1985). For example, the spontaneous limb movements produced by infants appear to be stereotypical, although qualitatively different from those exhibited by adults during purposeful reaching (Thelen, 1985; Thelen \& Fisher, 1983). Although the present findings of early articulatory stability correspond with the early motor development literature, they are at considerable odds with the most frequently reported finding in the speech development literature. In comparison with adult speakers, children consistently exhibit increased levels of variability in early articulatory performance (Goffman \& Smith, 1999; Green et al., 2000; Sharkey \& Folkins, 1985; Smith \& Gartenberg, 1984; Smith \& Goffman, 1998). These prior studies on the development of articulatory control have primarily studied children age 4 years and older. During this period of development, instability in articulatory movement patterns might be anticipated as children move away from "biologically preferred" articulatory patterns toward those required of their ambient language, whereas the stability observed in the mandibular movement at age 1 year in the present study may reflect a state of "prelinguistic equilibrium," with some oromotor movements being sharply constrained by biomechanic and neuromotor factors.

The difference reported across studies may also be related to differences among speech tasks and the kinematic signal's being used to represent articulatory movement. For instance, the present findings suggest that studies tracking the combined movement of lower lip and jaw would be expected to observe higher levels of variability in articulatory performance primarily because of the instability generated by the lower lip. In addition, because motor instability in early speech is probably closely tied to the difficulty of a speech utterance, a different picture of motor stability might have been observed in the present study if the subjects produced more complex speech stimuli. Clearly, the experimental tasks in the present study did not provide for a full test of the speech motor system, as the speech utterances were simplistic and already established within the child's repertoire.

\section{Some Aspects of Speech Motor Develop- ment Are Age-Specific}

Although the notion of motor milestones remains controversial in the motor development literature (e.g., Gottlieb, 1998; Newell, Scully, McDonald, \& Baillar- 
geon, 1990; Reed \& Bril, 1996; Thelen, 1995), several of the present experimental findings support the existence of age-based characteristics in articulatory performance. Young children's articulatory movements tended to be more similar to those of same-age peers than to those of adults, yielding higher coefficient values in the withinage comparison (Figure 5) than in the across-age comparison (Figure 4). Because within-subject stability was comparable or greater than within-age similarity for the lower lip, these age-based differences probably reflect qualitative differences in movement patterns rather than motor instability.

\section{Why Jaw vs. Tongue and Lips?}

In the following section, we briefly speculate about the maturational, biomechanic, and functional factors that could potentially establish the jaw as the most effective articulator for early speech. In early fetal development, the jaw's neuroanatomic infrastructure develops earlier than those supporting lip and tongue (Herring, 1985). For instance, while the lip musculature is still in the premyoblast stage at 8 weeks gestation (Gasser, 1967), the fetus is already opening the jaw (Humphrey, 1964). Young children may also rely on the jaw because its biomechanical architecture offers a source of movement stability. The mandible consists of a single bone that bilaterally articulates with the temporal bones and a network of symmetrical musculature (i.e., jaw depressors and elevators). In contrast, control over the interdigitated muscular layers that compose the highly deformable tongue and lips may require relatively greater constraint from the nervous system (Abbs, Gracco, \& Blair, 1984; Blair, 1986; Smith \& Kier, 1989). Thus, motor skill development may be a more protracted process for structures with greater degrees of freedom of control. Further research is needed to clarify the potential influence of these factors on shaping the course of speech motor development.

\section{Implications for the Development of Speech Abilities}

The present findings offer some limited predictions concerning the role of early coordinative biases on the sound-producing capabilities of the infant. If it is assumed that the young child adapts existing capabilities to approximate speech, then it is possible for well-established oromotor patterns to be contemporaneously advantageous for one phoneme while disadvantageous for another. For example, given the present findings we might deduce that the young child could successfully produce phonemes that can be effectively formed using the mandible as the primary mover (e.g., /b/), and be less able to produce those that tend to be associ- ated with graded lip control (e.g., /f/). Studies on early speech emergence support this prediction in that $/ \mathrm{f} /$ is rarely produced in early childhood and is not typically mastered until age 4 (Sanders, 1972), whereas bilabial stops are highly represented in early phonemic inventories (Stoel-Gammon, 1985). Thus, an initial step toward an improved understanding of speech development requires defining the characteristics of the immature oromotor system and assessing the compatibility of the existing coordinative organization with the spatial and temporal demands of individual phonemes and phoneme combinations.

\section{Methodological Issues}

Because the facial skin forms a tissue bridge between the lower lip and jaw, flesh-point tracking can provide only a gross estimate of independent jaw and lower lip motion. Using cineradiography, Kuehn and colleagues (Kuehn, Reich, \& Jordan, 1980) reported that the standard deviation between the vertical positions of a chin marker and a point identified on the mandibular bone is variable across subjects; it was reported to be as high as $1.28 \mathrm{~mm}$ during speech. These authors speculated that the lower lip pulling the skin overlying the chin accounted for some of this positional deviation. In the present investigation, a large amount of biomechanic coupling between the lower lip and jaw markers would have greatly diminished our ability to detect differences between their movement patterns across and within ages. Although it is likely that lower lip motions had some influence on our jaw signals, the large differences between lower lip and jaw performance during early childhood observed in the present study suggests that biomechanic coupling effects did not have a strong influence on the observed developmental trends. Future efforts will be directed toward quantifying potential positional errors associated with flesh-point tracking methods.

\section{Summary}

In summary, speech motor development entails the sequential emergence of articulatory control. In all the comparisons, the performance of the mandible exceeded the performance of the upper and lower lip in children 2 years old and younger.

The present findings suggest that the development of speech entails integrating the labial movements into this well-established mandibular movement pattern. Given these findings we posit that regularities across speakers in phonemic development and early articulatory strategies provide strong evidence for the significant influence of biomechanic and neuromotor biases on emerging speech motor skills. 


\section{Acknowledgments}

This research was supported in part by research grants (R01 DC00822 and R03 DC4643-01) from the National Institute on Deafness and Other Communication Disorders. The authors would like to thank Anne Smith for providing example interpolation routines and Gary Weismer and Ray Kent for helpful feedback on earlier versions of this manuscript.

\section{References}

Abbs, J. H. (1973). Some mechanical properties of lower lip movement during speech production. Phonetica, 28, 65-75.

Abbs, J. H., Gracco, V. L., \& Blair, C. (1984). Functional muscle partitioning during voluntary movement: Facial muscle activity for speech. Experimental Neurology, 85, 469-479.

Barlow, S. M., \& Farley, G. R. (1989). Neurophysiology of speech. In D. P. Kuehn, M. L. Lemme, \& J. M. Baumgartner (Eds.), Neural bases of speech, hearing, and language (pp. 146200). Boston: College-Hill Press.

Bekoff, A., \& Trainer, W. (1979). The development of interlimb co-ordination during swimming in postnatal rats. Journal of Experimental Biology, 83, 1-11.

Blair, C. (1986). Interdigitating muscle fibers throughout orbicularis oris inferior: Preliminary observations. Journal of Speech and Hearing Research, 29, 266-269.

Bosma, J. F. (1985). Postnatal ontogeny of performances of the pharynx, larynx, and mouth. American Review of Respiratory Disease, 131 (Suppl. 5), S10-S15.

Eguchi, S., \& Hirsh, I. J. (1969). Development of speech sounds in children. Acta Oto-Laryngologica (Suppl. 257), 1-51.

Gasser, R. F. (1967). The development of the facial muscles in man. American Journal of Anatomy, 120, 357-376.

Goffman, L., \& Smith, A. (1999). Development and phonetic differentiation of speech movement patterns. Journal of Experimental Psychology: Human Perception \& Performance, 25, 649-660.

Gottlieb, G. (1998). Myrtle McGraw's unrecognized conceptual contribution to developmental psychology. Developmental Review, 18, 437-448.

Green, J. R., Moore, C. A., Higashikawa, M., \& Steeve, R. W. (2000). The physiologic development of speech motor control: Lip and jaw coordination. Journal of Speech, Language, and Hearing Research, 43, 239-255.

Green, J. R., Moore, C. A., Ruark, J. L., Rodda, P. R., Morvee, W. T., \& VanWitzenburg, M. J. (1997). Development of chewing in children from 12 to 48 months: Longitudinal study of EMG patterns. Journal of Neurophysiology, 77, 2704-2727.

Hall, W. G., \& Oppenheim, R. W. (1987). Developmental psychobiology: Prenatal, perinatal, and early postnatal aspects of behavioral development. Annual Review of Psychology, 38, 91-128.

Hamburger, V., \& Oppenheim, R. (1967). Prehatching motility and hatching behavior in the chick. Journal of Experimental Zoology, 166, 171-203.
Hayden, D. A., \& Square, P. A. (1994). Motor speech treatment hierarchy: A systems approach. Clinics in Communication Disorders, 4, 151-161.

Herring, S. W. (1985). The ontogeny of mammalian mastication. American Zoology, 25, 339-349.

Humphrey, T. (1964). Some correlations between the appearance of human fetal reflexes and the development of the nervous system. Progress in Brain Research, 4, 93-135.

Humphrey, T. (1970). The development of human fetal activity and its relation to postnatal behavior. Advances in Child Development and Behavior, 5, 1-57.

Humphrey, T. (1971). Development of oral and facial motor mechanisms in human fetuses and their relation to craniofacial growth. Journal of Dental Research, 50, 1428-1441.

Kennedy, J. G. III, \& Kuehn, D. P. (1989). Neuroanatomy of speech. In D. P. Kuehn, M. L. Lemme, \& J. M. Baumgartner (Eds.), Neural bases of speech, hearing, and language (pp. 111145). Boston: College-Hill Press.

Kent, R. D. (1976). Anatomical and neuromuscular maturation of the speech mechanism: Evidence from acoustic studies. Journal of Speech and Hearing Research, 19, 421-447.

Kent, R. D. (1992). The biology of phonologic development. In C. A. Ferguson, L. Menn, \& C. Stoel-Gammon (Eds.), Phonological development: Models, research, and implications (pp 65-90). Timonium, MD: York Press.

Kent, R. D. (1999). Motor control: Neurophysiology and functional development. In A. Caruso \& E. Strand (Eds.), Clinical management of motor speech disorders in children (pp. 2971). New York: Thieme.

Kuehn, D. P., Reich, A. R., \& Jordan, J. E. (1980). A cineradiographic study of chin marker positioning: Implications for the strain gauge transduction of jaw movement. Journal of Acoustical Society of America, 67, 1825-1827.

Locke, J. L. (1983). Phonological acquisition and change. San Diego: Academic Press.

Locke, J. L. (1993). The child's path to spoken language (2nd ed.). Cambridge, MA: Harvard University Press.

MacNeilage, P. F. \& Davis, B. L. (1990a). Acquisition of speech production: Frames, then content. In M. Jeannerod (Ed.), Attention and performance XIII: Motor representation and control (pp. 453-476). Hillsdale, NJ: Lawrence Erlbaum.

MacNeilage, P. F. \& Davis, B. L. (1990b). Acquisition of speech production: The achievement of segmental independence. In W. J. Hardcastle \& A. Marchal (Eds.), Speech Production and Modeling (pp. 55-68). Dordrecht: Kluwer Academic.

MacNeilage, P. F., \& Davis, B. L. (2000). On the origin of internal structure of word forms. Science, 288, 527-531.

Matlab [Computer software]. (1999). Natick, MA: The MathWorks.

Meier, R., McGarvin, L., Zakia, R., \& Willerman, R. (1997). Silent mandibular oscillations in vocal babbling. Phonetica, 54, 153-171.

Moore, C. A., \& Ruark, J. L. (1996). Does speech emerge for earlier appearing oral motor behaviors? Journal of Speech and Hearing Research, 39, 1034-1047.

Motus [version 2, Computer software]. (1998). Englewood, CO: Peak Performance Technologies. 
Newell, K. M., Scully, D. M., McDonald, P. V., \& Baillargeon, R. (1990). Task constraints and infant grip configuration. Developmental Psychobiology, 22, 817-832.

Nittrouer, S. (1993) The emergence of mature gestural patterns is not uniform: Evidence from an acoustic study. Journal of Speech and Hearing Research, 36, 959-971.

Oller, D. K. (1980). The emergence of the sounds of speech in infancy. In G. H. Yeni-Komshian, C. A. Ferguson, \& J. Kavanagh (Eds.), Child phonology: Production (pp. 93112). New York: Academic Press.

Ostry, D. J., Vatikiotis-Bateson E., \& Gribble, P. L.(1997). An examination of the degrees of freedom of human jaw motion in speech and mastication. Journal of Speech, Language, $\mathcal{E}$ Hearing Research, 40, 1341-1351.

Preisser, D. A., Hodson, B. W., \& Paden, E. P. (1988). Developmental phonology: 18-29 months. Journal of Speech and Hearing Disorders, 53, 125-130.

Reed, E. S., \& Bril, B. (1996). The primacy of action in development. In M. L. Latash \& M. T. Turvey. (Eds.). Dexterity and its development. Mahwah, NJ: Lawrence Erlbaum Associates.

Sanders, E. K. (1972). When are speech sounds learned? Journal of Speech and Hearing Disorders, 37, 55-63.

Sharkey, S. G., \& Folkins, J. W. (1985). Variability of lip and jaw movements in children and adults: Implications for the development of speech motor control. Journal of Speech and Hearing Research, 28, 8-15.

Smith, A., \& Goffman, L. (1998). Stability and patterning of speech movement sequences in children and adults. Journal of Speech, Language, and Hearing Research, 41, 18-30.

Smith, A., Goffman, L., \& Stark, R. E. (1995). Speech motor development. Seminars in Speech and Language, 16, 87-99.

Smith, B. L. (1995).Variability of lip and jaw movements in the speech of children and adults. Phonetica, 52, 307-316.
Smith, B. L., \& Gartenberg, T. E. (1984). Initial observations concerning the developmental characteristics of labio-mandibular kinematics. Journal of the Acoustical Society of America, 75, 1599-1605.

Smith, K. K., \& Kier, W. M. (1989). Trunks, tongues, and tentacles: Moving with skeletons of muscles. American Scientist, 77, 29-35.

Stoel-Gammon, C. (1985). Phonetic inventories, 15-24 months: Longitudinal study. Journal of Speech and Hearing Research, $28,505-512$.

Sussman, H. M., Minifie, F. D., Buder, E. H., Stoel-Gammon, C., \& Smith, J. (1996). Consonant-vowel interdependencies in babbling and early words: Preliminary examination of a locus equation approach. Journal of Speech and Hearing Research, 39, 424-433.

Thelen, E. (1985). Developmental origins of motor coordination: Leg movements in human infants. Developmental Psychobiology, 18, 1-22.

Thelen, E. (1995). Motor development: A new synthesis. American Psychologist, 50, 79-95.

Thelen, E., \& Fisher, D. M. (1983). The organization of spontaneous leg movements in newborn infants. Journal of Motor Behavior, 15, 353-377.

Tingley, B. M., \& Allen, G. D. (1975). Development of speech timing control in children. Child Development, 46, 186-194.

Touwen, B. C. L. (1998). The brain and development of function. Developmental Review, 18, 504-526.

Vorperian, H. K., Kent, R. D., Gentry, L. R., \& Yandell, B. S. (1999). Magnetic resonance imaging procedures to study the concurrent anatomic development of vocal tract structures: Preliminary results. International Journal of Pediatric Otorhinolaryngology, 49, 197-206. 